\title{
Verlässliche Personenidentifizierung mittels Techniken der Künstlichen Intelligenz
}

\author{
Künstliche Intelligenz hält zunehmend Einzug in unseren Alltag, auch die \\ automatisierte Identifizierung natürlicher Personen wird davon erfasst. In diesem \\ Artikel werden die dabei entstehenden Risiken erläutert und erste Vorschläge \\ für die Zertifizierung geeigneter Sicherheitsmaßnahmen aufgezeigt.
}

\section{Einleitung}

Leonid Wolkow ist kein Unbekannter. Als Stabschef des zurzeit in einem Straflager inhaftierten Kremlgegners Alexej Nawalny hat er häufig Kontakt mit Medien und Politik, auch in sozialen Netzwerken ist er sehr aktiv. Dennoch sind mehrere Politiker auf einen Betrug hereingefallen: In einer Videokonferenz sprachen

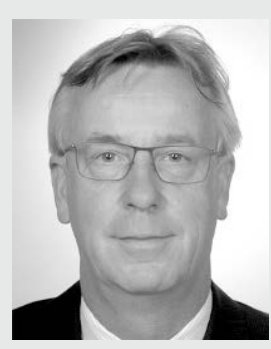

Dipl. Wirtsch.-Ing. Arno Fiedler

Der Geschäftsführer der Nimbus Technologieberatung $\mathrm{GmbH}$ ist seit 20 Jahren als Berater im Bereich des Identitätsmanagement und der Vertrauensdienste aktiv und engagiert sich ehrenamtlich als ETSI ESI ViceChair und als Vorstandsmitglied des Verbandes Sichere Digitale Identitäten e. V..

E-Mail: arno.fiedler@nimbus-berlin.com

\section{Prof. Dr. Christoph Thiel}

Professor für sichere und zuverlässige Softwaresysteme an der Fachhochschule Bielefeld, Standort Minden

Prof. Dr. Christian Thiel
Dozent für Wirtschaftsinformatik,
Institut für Informations und Prozess-
management
FHS St. Gallen, Hochschule für An-
gewandte Wissenschaften.
E-Mail: christian.thiel@bluewin.ch

sie nicht mit dem Nawalny-Mitarbeiter, sondern mit einem Betrüger, der sich für ihn ausgab. Im April 2021 haben unter anderem die niederländischen Zeitungen »de Volkskrant«, der »Guardian" und die "NL Times« berichtet, dass sich Politiker aus mehreren Ländern von einem falschen Wolkow haben täuschen lassen. Abgeordnete aus dem außenpolitischen Ausschuss der Niederlande sowie Politiker aus Großbritannien und verschiedenen baltischen Staaten hatten demnach mit dem vermeintlichen Wolkow gesprochen und erst später gemerkt oder die Information erhalten, dass sie einem Betrüger aufgesessen waren.

Weitere Aufmerksamkeit erhielten diese Medienberichte durch die zunächst aufgestellte Behauptung, der falsche Wolkow sei ein "Deep Fake« gewesen, ein durch Techniken der künstlichen Intelligenz (KI) erzeugtes Gesicht mit der Mimik und Stimme des Nawalny-Vertrauten, überzeugend genug, um in einer Videokonferenz von allen Teilnehmern als echt akzeptiert zu werden.

Allgemeiner formuliert steht hinter Deep Fake, abgeleitet von den Begriffen »Deep Learning" und »Fake», die Möglichkeit, Bild-, Video- oder Audiodaten so zu manipulieren, dass biometrische Merkmale wie Aussehen, Stimme oder auch charakteristische Gestik und Mimik von Personen täuschend echt imitiert werden. Auch wenn bisher kein Beweis veröffentlicht worden ist, dass der falsche Wolkow computergeneriert wurde - möglicherweise handelte es sich einfach nur um einen Doppelgänger, der von Maskenbildnern „klassisch“ hergerichtet wurde - zeigen diese Berichte, welche Qualität bereits heute Deep Fakes zugestanden wird. Tatsächlich wird die rasante Entwicklung der KI insbesondere im Bereich des Deep Learning dazu führen, dass Deep Fakes immer realistischer und einfacher zu generieren sein werden. In Zukunft werden automatisiert hergestellte hochwertige Fälschungen möglich und für jedermann zugänglich sein. Damit wird klar, welches destruktive Potential hinter Deep Fakes stecken kann: Prinzipiell können alle Attacken, die darauf basieren, dass sich ein Angreifer virtuell als eine andere Person ausgibt - zum Beispiel am Telefon, in einem Videochat oder einer Videokonferenz - durch die Deep Fake-Technologie vereinfacht und verstärkt werden. Dies gilt insbesondere auch für Angriffe gegen Verfahren der Remote-Identifizierung, die ohne die phy- 
sische Präsenz der zu prüfenden Person auskommen und stattdessen remote mittels Videoübertragung durchgeführt werden.

In den folgenden Abschnitten betrachten wir zunächst die grundlegenden Probleme und Risiken, die sich im Zusammenhang mit solchen Verfahren der Remote-Identifizierung ergeben. Wir diskutieren die aktuellen Rahmenbedingungen und Entwicklungen insbesondere hin zur automatisierten visuellen Identitätsprüfung bzw. des Identitätsnachweises. Die aktuellen Vorgaben klären allerdings nicht, wie die Verlässlichkeit der automatisierten Lösung bewertet werden kann. Implizit ist zumindest klar, dass es sich um KI-basierte Lösungen handeln wird. Wir betrachten daher die Möglichkeiten und Herausforderungen hinsichtlich der Standardisierung und Zertifizierung solcher Lösungen.

\section{Risiken}

Die Zunahme des digitalen Marktes hat die Idee der RemoteIdentifizierung einer Person attraktiv gemacht, da die RemoteIdentifizierung auch jenen Kunden, die physisch weit entfernt sind, den Zugang zu Identifizierungsdienstleistungen ermöglicht. Während der COVID-19-Pandemiekrise wurde die Möglichkeit, eine Person ohne physische Anwesenheit zu identifizieren, sogar noch wichtiger, da die physische Anwesenheit nicht nur umständlich ist, sondern sogar gefährlich sein kann, oder einfach nicht möglich ist, weil die Sicherheitsmaßnahmen zur Reduzierung der Auswirkungen der Pandemie eingehalten werden müssen. Die Möglichkeit des Remote-Identitätsnachweises fördert und erhöht die Möglichkeit elektronischer Transaktionen bei gleichzeitiger Sicherstellung der Gültigkeit der Identitäten der beteiligten Transaktionspartner.

Die Verfahren der Remote-Identifizierung lassen sich unterteilen in Verfahren, bei denen ein Live-Agent in Echtzeit mit der zu prüfenden Person in einem Videochat verbunden ist und in automatisierte Verfahren, bei denen in bzw. nach einer Videositzung die Identität ohne Personaleinsatz, also unbeaufsichtigt und automatisiert, überprüft und validiert wird. Entsprechend des Identitätsnachweisprozesses, wie er in [1] und in ähnlicher Form in \{2] skizziert wird, werden in beiden Fällen zusätzlich physische und / oder digitale Identitätsdokumente als Nachweis (Evidence) vorgezeigt.

Der Ansatz mit Live-Agenten kann zu einer schlechten Benutzererfahrung führen, wenn z.B. ein Kunde im Web-/Online-Kanal warten muss, bevor er mit dem Agenten verbunden werden kann. Außerdem führt der Personaleinsatz zu erheblichen Kosten für den Anbieter. Automatisierte Verfahren erfordern aber eventuell weitergehende Maßnahmen, um zusätzliche Risiken zu kompensieren. Gerade im digitalen Umfeld ist aufgrund des fehlenden direkten persönlichen Kontakts und dem Wegfall der Anreise die Hemmschwelle für Missbrauchsversuche herabgesetzt.

Entsprechend wichtig ist es für Hersteller bzw. Betreiber entsprechender Services eine Risikobewertung durchführen, um sicherzustellen, dass das Sicherheitsniveau dem Grad des Risikos angemessen ist, und um die Auswirkungen von Sicherheitsvorfällen zu minimieren. Derzeit gibt es in der aktuellen Literatur nur wenige Quellen zu Bedrohungen und Schwachstellen bzw. Verletzlichkeiten und weitergehend zu Risiken hinsichtlich von Verfahren der Remote-Identifizierung (s. [1], [2]). So empfiehlt [1] einheitliche Risikoanalysen, die systematisch durchgeführt werden sollten, um einen sicheren Nachweisprozess zu gewährleis- ten, die verschiedenen Implementierungen abzustimmen und zu vergleichbaren Ergebnissen zu kommen. Zudem sollte eine regelmäßige Überprüfung der Risiken und ein Austausch von Sicherheitsvorfällen zwischen den verschiedenen Akteuren die Konvergenz für Prozesse mit vergleichbaren Vertrauensstufen (s. Levels of Assurance (LoAs), Artikel 8 aus [5]) weiter stärken. Eine solche Risikoanalyse kann sich beispielsweise auf die ISO/IEC 27005 abstützen. Hinsichtlich konkreter Einzelrisiken müssen dabei spezifische Risikoszenarien für Verfahren der RemoteIdentifizierung betrachtet werden. In einem Button-Up-Ansatz bewegt man sich entlang der Phasen des Identitätsnachweisverfahren und identifiziert dort Bedrohungen gegen primäre und sekundäre Assets (Vorgehen nach ISO/IEC 27005) sowie mögliche Schwachstellen / Verletzlichkeiten.

Der wichtigste identifizierte Asset im Kontext der Identitätsprüfung ist der Identitätsprüfungsprozess selbst und alle im Rahmen dieses Prozesses verarbeiteten Nachweise. Verarbeitete und gespeicherte Daten und Nachweise werden ebenfalls als primäre Assets anerkannt. Beispiele für diese Assets sind private Daten des Antragstellers, Fotos (Scans), Videoaufzeichnungen, Daten, die der Antragsteller im Rahmen des Einleitungsprozesses übermittelt hat, Daten, die der Antragsteller als Ergebnis erhält sowie Identitätsnachweise.

Der Identitätsnachweisprozess basiert zudem auf vielen unterstützenden sekundären Assets, die ebenfalls berücksichtigt werden sollten, insbesondere die für die Durchführung des Prozesses verwendeten Computersysteme einschließlich Netzwerk und Software, der Bedienerarbeitsplatz, ausgestattet mit Kamera und Sprachgeräten, der PC oder das mobile Gerät der zu prüfenden Person, Kommunikationskanäle, interne Datenbanken (z. B. Protokolle, Archive), externe Register (z. B. Register gestohlener Ausweisdokumente) und unterstützende Dienste von externen Anbietern (z. B. Zertifikatsvalidierungsdienst).

Die für ein Identitätsnachweisverfahren spezifischen Risiken fallen in zwei Hauptkategorien von Bedrohungen (vgl. [1]) nämlich:

- Gefälschte Nachweise: Ein Antragsteller behauptet eine falsche Identität unter Verwendung gefälschter Nachweise.

- Identitätsdiebstahl: Ein Antragsteller verwendet gültige Nachweise, die einer anderen Person zugeordnet sind.

Der ersten Bedrohungskategorie wird durch Attribut- und Nachweisvalidierung begegnet, während der zweiten durch Bindung an den Antragsteller begegnet wird.

Die entlang des Identitätsnachweisprozesses identifizierten Gefährdungen (Bedrohungen und Schwachstellen) können demnach folgenden Risikodimensionen zugeordnet werden.

1. Der Identitätsnachweisprozess kann durch gefälschte und/oder manipulierte Nachweise (Evidenz) kompromittiert werden.

2. Der Identitätsnachweisprozess kann durch die Verwendung von Nachweisen kompromittiert werden, die gekündigt, widerrufen oder verloren/gestohlen wurden

3. Der Identitätsnachweisprozess kann durch einen Betrüger kompromittiert werden, der die legitime Identität einer anderen Person vorgibt

4. Der Identitätsnachweisprozess kann durch Manipulation von Bilderfassungssystemen oder Übertragungskanälen (bei Remote-Identitätsnachweis) beeinträchtigt werden

Um einzelne Risiken besser behandeln zu können empfiehlt es sich konkrete Gefährdungsszenarien zu beschreiben. Viele Szenarien sind für jedes Identifikationsnachweisverfahren (sei es 
Vor-Ort beim Dienstleister, Online in Echtzeit mit einem LiveAgenten oder vollständig automatisiert) relevant.

Wir konzentrieren uns hier zunächst auf die spezifischen Angriffe auf automatisierte Identitätsnachweisverfahren. Diese lassen sich in technische Angriffe und prozedurale / prozessorientierte Angriffe unterteilen (vgl. [1]).

Technische Angriffe richten sich auf die Backend-Systeme oder einzelne ihrer Komponenten, auf die Datenübertragung (Manin-the-Middle-Angriffe und Angriffe gegen die Verschlüsselung), oder auf das Benutzergerät des realen Benutzers. Dabei sind die Angriffe nicht auf den Einsatz dieser Assets beschränkt, sondern auf den gesamten Life Cycle. Z.B. kann ein Angreifer versuchen, bereits den Software-Entwicklungsprozess anzugreifen und so eine Backdoor in eine Komponente vor deren Nutzung einzuschleusen. Zunehmend werden für die Backendsysteme moderne KI-Systeme genutzt, die anfällig gegenüber qualitativ neuartigen Angriffen, die wir analog zu [6] KI-spezifische Angriffe nennen.

Diese Angriffe sind z. T. bereits durch legitime Anfragen an das KI-Modell, d.h. durch die "normale" Nutzeraktion mit dem Backend, möglich. Die für Remote-Identifizierungsverfahren relevantesten Angriffe dieser Art sind:

- Evasion/Adversarial Attacks: Durch eine Manipulation von Eingabedaten verleiten Angreifer das KI-Modell im Betrieb zu vom Entwickler nicht vorgesehenen Ausgaben bzw. Aktionen.
Das Modell selbst wird hierbei nicht verändert. Bereits geringfügige Änderungen der Eingabedaten, die schwierig zu detektieren und selbst für Menschen nicht unmittelbar erkennbar sind oder von ihnen als irrelevant interpretiert werden, können bedeutsame Auswirkungen haben.

- Data Poisoning Attacks: Ein Angreifer könnte versuchen Trainingsdaten für die benutzen KI-Systeme vorab zu manipulieren oder eigene einzuschleusen und das Verhalten der Systeme in seinem Sinne zu beeinflussen. Aufgrund der vielen Daten und der mangelnden Transparenz sind diese Angriffe meist schwer detektierbar.

- Model Stealing Attacks: Angreifer extrahieren die Funktionalität des Modells. Dabei werden Informationen über die Struktur des Modells, z. B. relevante Entscheidungsparameter, extrahiert oder die Funktionalität des angegriffenen Modells (näherungsweise) kopiert. Ziel ist die Vorbereitung anderer Angriffe, die die Kenntnis dieses Modells erfordern.

Bei prozeduralen / prozessorientierten Angriffe versucht der Angreifer / Betrüger den Identitätsnachweisprozess an sich zu kompromittieren, indem er die legitime Identität einer anderen Person (z. B. durch Deep Fakes) vorgibt, durch Social Engineering Angriffe, durch das Ausnutzen konkreter Prozessfehler bis hin durch das Zwingen einer anderen Person, eine Identifizierung durchzuführen. Dies kann in Form von körperlicher Gewalt, physische

\title{
Springer Vieweg
}

\section{Strategien in der Informationstechnik}

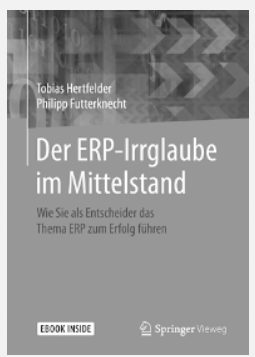

\author{
T. Hertfelder, P. Futterknecht \\ Der ERP-Irrglaube im Mittelstand \\ Wie Sie als Entscheider das Thema ERP \\ zum Erfolg führen \\ 2019, XI, 188 S. 100 Abb. Book + eBook. Brosch. \\ $€$ (D) $39,99|€(A) 41,86|{ }^{*} \mathrm{CHF} 44.50$ \\ ISBN 978-3-662-59142-0 \\ $€ 29,99 \mid{ }^{*} \mathrm{CHF} 35.50$ \\ ISBN 978-3-662-59143-7 (eBook)
}

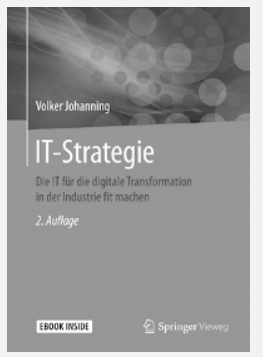

\author{
V. Johanning \\ IT-Strategie \\ Die IT für die digitale Transformation in der \\ Industrie fit machen \\ 2., Akt. u. erw. Aufl. 2019, XV, 312 S. 149 Abb., \\ 36 Abb. in Farbe. Book + eBook. Geb. \\ $€$ (D) $39,99|€(A) 41,86|{ }^{*} \mathrm{CHF} 44.50$ \\ ISBN 978-3-658-26489-5 \\ $€ 29,99 \mid{ }^{*} \mathrm{CHF} 35.50$ \\ ISBN 978-3-658-26490-1 (eBook)
}

\section{Ihre Vorteile in unserem Online Shop: \\ Über 280.000 Titel aus allen Fachgebieten | eBooks sind auf allen Endgeräten nutzbar | Kostenloser Versand für Printbücher weltweit}

$€(D)$ : gebundener Ladenpreis in Deutschland, $€(A)$ : in Österreich. * : unverbindliche Preisempfehlung. Alle Preise inkl. MwSt. 
Gewalt gegen Angehörige, Erpressung, etc. erfolgen. Die Szenarien unterscheiden sich hinsichtlich der Schwierigkeit, den Angriff durchzuführen und den dazu benötigten Ressourcen, was bei der Risikobewertung im Einzelfall zu berücksichtigen ist.

Neben vorsätzlichen Angriffen sind weitere Gefährdungen zu betrachten.

- Moderne KI-Systeme sind in der Lage, aus riesigen Datenmengen zu lernen und auf diesen «Erfahrungen» basierend Entscheidungen «intuitiv» zu treffen. Das bedeutet, dass ein KISystem nicht wie klassische Systeme nur detaillierte, vorab geschriebene Anweisungen ausführen, sondern in der Lage ist, dynamische Lösungen für Probleme zu finden, die auf Korrelationen in den Daten basieren. Insbesondere weiterlernende Systeme entwickeln sich im laufenden Betrieb (eventuell auch unvorhersehbar) kontinuierlich und selbstständig weiter. Selbst wenn ein KI-System nach Abschluss einer vorgegebenen Lernund Testphase nicht mehr angepasst wird (also nicht mehr weiterlernt), kann sich die Betriebsumgebung (concept drift) und damit sein Verhalten ggf. zum Schlechteren hin ändern.

- Bei einigen KI-Methoden ist im Gegensatz zu klassischen Algorithmen nicht mehr nachvollziehbar, wieso ein KI-System bei einer konkreten Fragestellung zu einer bestimmten Antwort gelangt ist (Black-Box-Problem). Die Ansätze der künstlichen Intelligenz sind zudem mit bekannten Problemen der Statistik konfrontiert, so dass die von einem KI-System ausgegebenen Resultate unter Umständen fehlerhaft sein können, Scheinkausalitäten erzeugt werden oder es gar zu systematischen Fehler kommt.

- Überdies sind die Systeme von der Qualität der Daten und Algorithmen abhängig. So können systematische Fehler in Daten oder Algorithmen (z.B. versteckte Einseitigkeiten [Bias], etwa, wenn in Daten eine Bevölkerungsgruppe über- oder unterrepräsentiert ist) angesichts der Größe und Komplexität der verwendeten Daten oft nicht erkannt werden.

\section{Rahmenbedingungen}

Entsprechend ihrer großen Bedeutung hat die EU-Kommission die elektronische Identifizierung als Voraussetzung der Nutzung von Vertrauensdiensten zu einem zentralen Element der "Verordnung über elektronische Identifizierung und elektronische Vertrauensdienste (eIDAS)" (s. [5]) gemacht. Bevor Vertrauensdienste genutzt werden können, muss der potenzielle Nutzer (der Antragsteller) eindeutig identifiziert werden. Nur somit kann sichergestellt werden, dass Vertrauensdienste nur diejenigen Anwender erhalten, die dazu auch tatsächlich berechtigt sind. eIDAS definiert u.a. die Identifizierungs-Anforderungen, die Vertrauensdiensteanbieter mit dem höchsten Sicherheitsniveau - sogenannte qualifizierte Vertrauensdiensteanbieter - erfüllen müssen.

eIDAS sieht in Art. 24 vor, dass eine Identitätsprüfung vor Ort durchgeführt werden muss oder eine der Vor-Ort-Prüfung gleichwertige Prüfung der Identität erforderlich ist. Ob eine Technologie diese Anforderungen erfüllt, wird von einer akkreditierten Konformitätsbewertungsstelle festgestellt. Auf diese Weise kann die Identifizierung grundsätzlich durch persönliche Anwesenheit oder aus der Ferne (remote) mittels elektronischer Identifizierungsmittel durchgeführt werden. Möglich sind auch andere Identifizierungsmethoden, vorausgesetzt, sie sind auf nationa- ler Ebene anerkannt und bieten eine der Anwesenheit vergleichbare Sicherheit.

Hierzu fehlten in Deutschland bisher an automatische RemoteIdentifizierungsverfahren angepasste Prüfkriterien.

Die Bundesnetzagentur hat Anfang April 2021 im Einvernehmen mit dem Bundesamt für Sicherheit in der Informationstechnik (BSI) und nach Anhörung des Bundesbeauftragten für den Datenschutz und der Informationsfreiheit offizielle Kriterien für die automatisierte optische Identitätsprüfung (Identifizierung einer natürlichen Person unter Nutzung einer Videoidentifizierung mit einem automatisierten Verfahren, [3]) vorgelegt. Bislang gab es solche Kriterien lediglich für Video-Ident-Verfahren, bei denen die Verifizierung durch einen menschlichen Prüfer, also einen Live-Agenten, stattfindet. Grundlage für den neuen Kriterienkatalog ist das deutsche Vertrauensdienstegesetz, das die nationale Umsetzung der EU-weiten eIDAS-Verordnung in deutsches Recht darstellt [4]. Der nun von der Bundesnetzagentur und dem BSI veröffentlichte Kriterienkatalog schreibt für die automatisierte Identitätsprüfung u.a. folgende Anforderungen vor.

- Durchführung der Videoidentifizierung in Echtzeit und ohne Unterbrechung

- Ende-zu-Ende-Verschlüsselung der audiovisuellen Kommunikation zwischen der zur Videoidentifizierung mit automatisiertem Verfahren genutzten IT-Technik und der zu identifizierenden Person

- ausreichende Bild- und Tonqualität

- geeignete Maßnahmen zur Überprüfung der Aktualität des Vorganges

- ausschließliche Zulässigkeit von fälschungssicheren Identitätsdokumenten mit prüfbaren Sicherheitsmerkmalen als Nachweis

- geeignete Maßnahmen zur Verhinderung von Manipulation bei Videobild, des ID-Dokumentes und der Person, insbesondere durch technische Maßnahmen, die eine Interaktion der zu prüfenden Person mit dem IT-System bzw. eine dabei stattfindende Manipulation erkennbar machen

- technische Maßnahmen zur Erkennung der Manipulation des Videobildes

- ausdrückliche Einwilligung der zu prüfenden Person zur Aufzeichnung des Identifizierungsprozess, ausführliche Datenschutzbelehrung

- Einhaltung der Speicherfristen nach eIDAS

- Schwärzung von für die Identifikation nicht erforderlichen Daten

- Mindestdauer von 15 Sekunden der (aufgenommenen) Videosequenz

- Möglichkeit der IT gestützten Auswertung der Anforderungen an Aufzeichnung und Aufbewahrung sowie Freigabe

- Meldung von Betrugsversuchen an die BNetzA

- Bestätigung der Umsetzung dieser Anforderungen durch eine Konformitätsbewertungsstelle.

Die Identifizierung gilt nicht für das Ausstellen qualifizierter Zertifikate für die Website Authentifizierung und ist auf das Ausstellen so genannte Ad-hoc Zertifikate beschränkt. 


\section{Herausforderungen hinsichtlich Standardisierung und Zertifizierung}

Tatsächlich lassen diese notwendigen und sinnvollen Anforderungen aus [3] einen deutlichen Interpretations- und Gestaltungsraum, so dass im konkreten Fall einer Identifizierungslösung weitere Detaillierungen notwendig sind. Insbesondere sollten spezifischere Parameter für die Eignung und Angemessenheit von Maßnahmen vorgegeben werden. Damit stößt man zwangsläufig auf das Problem der Verlässlichkeit der zur Automatisierung genutzten Verfahren. Hier sind die Aspekte der automatischen Erkennung von Manipulationsversuchen (z.B. Manipulation des Videobildes und -tones) und der zeitlichen Aktualität des Identifizierungsvorganges zu beachten. Diese setzen zunehmend auf moderne KI-Systeme, und für diese ist nach unseren obigen Betrachtungen (Abschnitt 2 und 3) im Kern offen, wie eine entsprechende Sicherheit nachvollziehbar nachgewiesen werden kann. Es ist kaum vorstellbar, dass dieses ohne eine Zertifizierung entsprechend eines noch festzulegenden Standards denkbar ist. Auch aus Sicht des BSI (s. [6]) besteht ein Handlungsbedarf zur Entwicklung von Standards, technischen Richtlinien, Prüfkriterien und Prüfmethoden, um die Sicherheit für KI-Systeme für kritische Anwendungskontexte (insbesondere den Kontext der automatisierten Identifizierung) verlässlich zu bewerten und technisch zu prüfen. Auch für weniger kritische Anwendungen fehlen (mit wenigen Ausnahmen) Maßstäbe für die Sicherheit.

Unabhängig von ggf. noch zu definierenden Einzelmaßnahmen lassen sich für den übergreifenden Zertifizierungsprozess bereits jetzt folgende Ansatzpunkte finden:

- Eine Zertifizierung sollte durchgeführt werden, bevor das KISystem in der Praxis zum Einsatz kommt.

- Das KI-System wird unter Umständen einige Zeit nach seiner Inbetriebnahme den Kriterien der Zertifizierung nicht mehr gerecht. Deshalb sollen KI-Systeme in regelmäßigen Abständen rezertifiziert werden.

- Andererseits sollte vermieden werden, das System nach jedem Update oder jeder Weiterentwicklung rezertifizieren zu müssen.

- Systemgrenzen, Detailgrad und Prüftiefe bei der Zertifizierung und Rezertifizierung sollten sich an einer Risikoabschätzung des KI-Systems in seinem Anwendungsgebiet orientieren - je höher die Kritikalität im Anwendungskontext eingeschätzt wird, desto umfangreicher sollten der Detailgrad und die Prüftiefe der Zertifizierung ausfallen.

- Um zudem auf technologische Weiterentwicklungen reagieren zu können sollten die Zertifizierung ein dauerhaft offener Prozess sein und beim Einsatz, Betrieb und Weiterentwicklung des KI-Systems insbesondere die Aspekte der kontinuierlichen Verbesserung berücksichtigt werden.

- Zudem ist es hilfreich, die Prüfverfahren zu dokumentieren, sodass Erfahrungswerte gesammelt und neue Tendenzen und Entwicklungen frühzeitig identifiziert werden können.

- Da die eIDAS Verordnung die europaweite Freizügigkeit von Identifizierungsdiensten ermöglicht, ist es geboten, dass auch die Standards und Kriterien für die Zertifizierung vereinheitlich werden, ein erstes Beispiel bilden hierfür die Bemühungen von ETSI ESI in der TR 119461. [9]

\section{Ausblick}

Im Vergleich zu bisherigen Standards im Umfeld der Identitätsprüfung und -nachweise ([7], [8]) erscheint das grundlegende Problem im Zusammenhang mit der automatisierten Identifizierung zu sein, dass die dabei genutzten Techniken der künstlichen Intelligenz bei weitem noch nicht so gut verstanden werden wie klassische Techniken wie z.B. kryptographische Verfahren, Chipkarten etc. Das Black-Box-Problem beeinflusst die IT-Sicherheit und das Vertrauen in das Identifizierungsverfahren maßgeblich.

Beispielsweise ist es bei komplexeren KI-Systemen häufig schwierig Auswirkungen von möglichen Angriffen vom statistisch bedingten Fehlverhalten der KI-Systeme zu unterscheiden, so dass ggf. Angriffe unerkannt bleiben oder es umgekehrt zu unberechtigten Alarmen kommt.

Das Vertrauen in ein System, dessen Funktionsweise und einzelne Ausgaben bzw. Aktionen nicht vollständig verstanden oder erklärt werden kann, bleibt oft hinter dem Vertrauen gegenüber einem menschlichen Kontakt zurück. Somit besteht Anlass zu der Sorge, dass KI-gestützte Verfahren, welche für »Deep Fakes« verwendet werden, erfolgreicher zum Einsatz kommen als die Verfahren, welche für eine remote-Identifizierung verwendet werden.

\section{Literatur}

[1] Draft ETSI TS 119461 V0.0.6d (2021-04), Electronic Signatures and Infrastructures (ESI); Policy and security requirements for trust service components providing identity proofing of trust service subjects, 2021.

[2] REMOTE ID PROOFING Analysis of methods to carry out identity proofing remotely, ENISA, März 2021.

[3] Vorläufige Anerkennung einer innovativen Identifizierungsmethode gemäß § 11 Absatz 3 VDG, 01.04.2021.

[4] Vertrauensdienstegesetz (VDG), 18.07.2017.

[5] REGULATION (EU) No 910/2014 OF THE EUROPEAN PARLIAMENT AND OF THE COUNCIL of 23 July 2014 on electronic identification and trust services for electronic transactions in the internal market and repealing Directive 1999/93/EC[6] Mandate M460: "Standardisation Mandate to the European Standardisation Organisations CEN, CENELEC and ETSI in the Field of Information and Communication Technologies Applied to Electronic Signatures".

[6] Sicherer, robuster und nachvollziehbarer Einsatz von KI, Bundesamt für Sicherheit in der Informationstechnik, 2021.

[7] Technische Richtlinie TR-03147 Vertrauensniveaubewertung von Verfahren zur Identitätsprüfung natürlicher Personen, Bundesamt für Sicherheit in der Informationstechnik, 2021.

[8] ETSI TR 119460 Electronic Signatures and Infrastructures (ESI); Survey of technologies and regulatory requirements for identity proofing for trust service subjects.

[9] ETSI TR 119461 Electronic Signatures and Infrastructures (ESI); Policy and security requirements for trust service components providing identity proofing of trust service subjects 